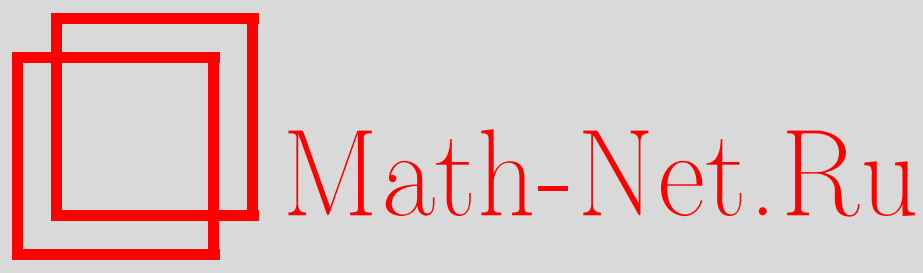

В. Розенхаус, Бесконечные законы сохранения для дифференциальных систем, ТМФ, 2009, том 160, номер 1, 202-210

DOI: https://doi.org/10.4213/tmf6391

Использование Общероссийского математического портала Math-Net.Ru подразумевает, что вы прочитали и согласны с пользовательским соглашением http://www . mathnet.ru/rus/agreement

Параметры загрузки:

IP : 52.6 .47 .48

26 апреля 2023 г., $12: 43: 42$

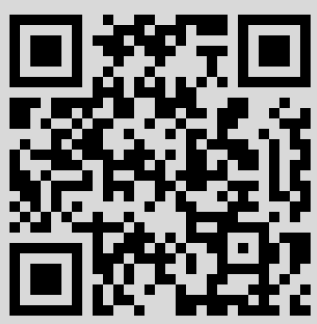




\title{
БЕСКОНЕЧНЫЕ ЗАКОНЫ СОХРАНЕНИЯ ДЛЯ ДИФФЕРЕНЦИАЛЬНЫХ СИСТЕМ
}

\begin{abstract}
Исследуются лагранжевы системы дифференциальных уравнений в частных производных, которые допускают бесконечномерные алгебры симметрий, параметризованные произвольными функциями от зависимых переменных. Показано, что эти системы обладают бесконечными наборами существенных законов сохранения.
\end{abstract}

Ключевые слова: бесконечная симметрия, закон сохранения.

\section{1. ВВЕДЕНИЕ}

Соотношение между группами вариационных симметрий и законами сохранения имеет давнюю историю и восходит к классическим результатам Нётер [1] (см. также монографию [2]); $r$-параметрическим группам симметрий Ли и соответствующим законам сохранения были посвящены многие работы (см., например, [3]-[13], а также [14]). В то же время бесконечные алгебры симметрий и их связи с законами сохранения были изучены существенно меньше. Согласно второй теореме Нётер [1] бесконечные вариационные симметрии с произвольными функциями от всех независимых переменных приводят не к законам сохранения, а к определенному соотношению между уравнениями исходной дифференциальной системы (что означает, что система является недоопределенной). Было показано, что бесконечные вариационные симметрии с произвольными функциями не от всех независимых переменных приводят к конечному числу существенных локальных законов сохранения [15], при этом каждый существенный закон сохранения определен конкретным видом граничных условий (см., например, работы [16]-[18]). В работе [19] был отмечен существенно иной случай, когда бесконечные симметрии, параметризованные произвольными функциями от зависимых переменных, приводят к бесконечному набору существенных законов сохранения (локальных сохраняющихся плотностей). Известный пример подобной ситуации дается уравнениями типа уравнения Лиувилля (см. статьи [20]-[22]), которые можно проинтегрировать с помощью метода Дарбу [23], [24]. Случай одного скалярного уравнения второго порядка, допускающего бесконечные вариационные симметрии с произвольными функциями от

${ }^{*}$ Department of Mathematics and Statistics, California State University, Chico, USA. E-mail: vrosenhaus@csuchico.edu 
зависимой переменной $u(u=u(x, t))$ и ее первых производных $u_{x}, u_{t}$, исследовался в работе [25], где были найдены классы дифференциальных уравнений в частных производных, обладающих бесконечными симметриями, а также соответствующие бесконечные наборы существенных сохраняющихся величин.

Настоящая работа является обобщением работы [25] на случай систем из двух уравнений с двумя зависимыми переменными. Мы исследуем существенные законы сохранения, соответствующие бесконечным вариационным симметриям, которые параметризованы произвольными функциями от зависимых переменных. При этом мы следуем идеологии, сформулированной в работе [25], и показываем, что аналогично случаю единственной зависимой переменной бесконечные симметрии таких дифференциальных систем действительно приводят к бесконечному набору существенных локальных законов сохранения.

Мы будем рассматривать задачу поиска систем из двух дифференциальных уравнений первого порядка с двумя зависимыми и двумя независимыми переменными $(u=u(x, t), v=v(x, t))$, допускающих бесконечные вариационные симметрии с произвольными функциями от переменных $u$ и $v$. Мы приведем примеры классов таких систем и соответствующих сохраняющихся величин.

\section{2. БЕСКОНЕЧНЫЕ СИММЕТРИИ И СУЩЕСТВЕННЫЕ ЗАКОНЫ СОХРАНЕНИЯ}

Кратко опишем подход, которому мы будем следовать; подробности можно найти в работах [15], [25]. Под законом сохранения для дифференциальной системы

$$
\omega^{a}\left(x, u, u_{i}, \ldots\right)=0, \quad i=1, \ldots, m+1, \quad a=1, \ldots, n,
$$

где $u_{i}^{a} \equiv \partial u^{a} / \partial x^{i}, x^{i}=x^{1}, x^{2}, \ldots, x^{m}, t$, понимается уравнение непрерывности

$$
D_{i} K_{i} \doteq 0, \quad K_{i}=K_{i}\left(x_{j}, u^{a}, u_{j}, \ldots\right), \quad i, j=1, \ldots, m+1, \quad a=1, \ldots, n
$$

$\left(K_{i}\right.$ - гладкие функции), которое выполнено для любых решений исходной системы (в этом смысле мы используем знак $\doteq$ ). Каждый закон сохранения определен с точностью до преобразования эквивалентности $K_{i} \rightarrow K_{i}+P_{i}$, где $D_{i} P_{i} \doteq 0$ [2]. Два закона сохранения принадлежат одному и тому же классу эквивалентности, если они отличаются на тривиальный закон сохранения. Закон сохранения $D_{i} K_{i} \doteq 0$ является тривиальным, если $K_{i}$ обращается в нуль на решениях, $K_{i} \doteq 0, i=1, \ldots, m+1$, или если уравнение непрерывности выполнено во всем пространстве, $D_{i} K_{i}=0$ [2] (тривиальность второго типа можно сформулировать в терминах замкнутых дифференциальных форм [26]).

Под существенным законом сохранения [15] понимается такой нетривиальный закон сохранения $D_{i} K_{i} \doteq 0$, который приводит к ненулевой сохраняющейся величине:

$$
D_{t} \int_{D} K_{t} d x^{1} d x^{2} \ldots d x^{m} \doteq 0, \quad x \in D \subset \mathbb{R}^{m+1}, \quad K_{t} \neq 0 .
$$

Рассмотрим функции $u^{a}=u^{a}(x)$, определенные на области $D$ в $(m+1)$-мерном пространстве-времени. Пусть

$$
S=\int_{D} L\left(x^{i}, u^{a}, u_{i}^{a}, \ldots\right) d^{m+1} x
$$


- функционал действия с плотностью лагранжиана $L$. Тогда уравнения движения имеют вид

$$
E^{a}(L) \equiv \omega^{a}\left(x, u, u_{i}, u_{i j}, \ldots\right)=0, \quad a=1, \ldots, n, \quad i, j=1, \ldots, m+1,
$$

где

$$
E^{a}=\frac{\partial}{\partial u^{a}}-\sum_{i} D_{i} \frac{\partial}{\partial u_{i}^{a}}+\sum_{i \leqslant j} D_{i} D_{j} \frac{\partial}{\partial u_{i j}^{a}}+\cdots
$$

- оператор Эйлера-Лагранжа.

Рассмотрим инфинитезимальное преобразование с каноническим оператором

$$
X_{\alpha}=\alpha^{a} \frac{\partial}{\partial u^{a}}+\sum_{i}\left(D_{i} \alpha^{a}\right) \frac{\partial}{\partial u_{i}^{a}}+\sum_{i \leqslant j}\left(D_{i} D_{j} \alpha^{a}\right) \frac{\partial}{\partial u_{i j}^{a}}+\cdots
$$

где $\alpha^{a}=\alpha^{a}\left(x, u, u_{i}, \ldots\right), i, j=1, \ldots, m+1, a=1, \ldots, n$. Вариация функционала $S$ при преобразовании под действием оператора $X_{\alpha}$ имеет вид

$$
\delta S=\int_{D} X_{\alpha} L d^{m+1} x
$$

Оператор $X_{\alpha}$ является вариационной (нётеровской) симметрией, если

$$
X_{\alpha} L=D_{i} M_{i}, \quad M_{i}=M_{i}\left(x, u, u_{i}, \ldots\right), \quad i=1, \ldots, m+1,
$$

где $M_{i}$ - гладкие функции. Тождество Нётер [27] (см. также работы [15] или [10] по поводу используемого здесь варианта тождества) связывает оператор $X_{\alpha}$ с оператором $E^{a}$ :

$$
X_{\alpha}=\alpha^{a} E^{a}+D_{i} R_{\alpha i}, \quad i=1, \ldots, m+1, \quad a=1, \ldots, n,
$$

где

$$
R_{\alpha i}=\alpha^{a} \frac{\partial}{\partial u_{i}^{a}}+\left\{\sum_{k \geqslant i}\left(D_{k} \alpha^{a}\right)-\alpha^{a} \sum_{k \leqslant i} D_{k}\right\} \frac{\partial}{\partial u_{i k}^{a}}+\cdots
$$

Применяя к $L$ тождество (7) и используя (6) и (8), получаем

$$
D_{i}\left(M_{i}-R_{\alpha i} L\right)=\alpha^{a} \omega^{a}, \quad i=1, \ldots, m+1, \quad a=1, \ldots, n,
$$

что на многообразии решений $\left(\omega=0, D_{i} \omega=0, \ldots\right)$

$$
D_{i}\left(M_{i}-R_{\alpha i} L\right) \doteq 0, \quad i=1, \ldots, m+1
$$

приводит к первой теореме Нётер: любое преобразование однопараметрической вариационной симметрии $X_{\alpha}(4)$ порождает закон сохранения (10). Теперь рассмотрим бесконечные вариационные симметрии. 
2.1. Произвольные функции независимых переменных. Во второй теореме Нётер [1] рассматриваются группы бесконечных вариационных симметрий в случае, когда вектор симметрии $\alpha$ имеет вид

$$
\alpha^{a}=l^{a} p(x)+b_{i}^{a} D_{i} p(x)+c_{i j}^{a} D_{i} D_{j} p(x)+\cdots,
$$

где $p(x)$ - произвольная гладкая функция от всех базисных пространственных переменных. Случай $p(x)$, являющейся произвольной функцией не от всех базисных переменных, исследовался в работе [15]. Для нётеровского преобразования симметрии $X_{\alpha}(4)$ в этом случае имеем

$$
\delta S=\int_{D} \delta L d^{m+1} x=\int_{D} X_{\alpha} L d^{m+1} x=\int_{D} D_{i} M_{i} d^{m+1} x=0, \quad x \in D,
$$

поэтому должны выполняться следующие условия на $M_{i}$ (нётеровские граничные условия) [15]:

$$
\left.M_{i}(x, u, \ldots)\right|_{x^{i} \rightarrow \partial D}=0, \quad i=1, \ldots, m+1 .
$$

Уравнения (13) обычно удовлетворяются при "регулярном" асимптотическом поведении функции $u$, т.е. когда $u^{a}, u_{i}^{a} \rightarrow 0$ при $x \rightarrow \pm \infty$, или для периодических решений. Интегрируя уравнение (10) по пространству переменных $x^{1}, x^{2}, \ldots, x^{m}$, получаем

$$
\int_{D} D_{t}\left(M_{t}-R_{\alpha t} L\right) d x^{1} d x^{2} \ldots d x^{m} \doteq \int_{D} \sum_{i=1}^{m} D_{i}\left(R_{\alpha i} L-M_{i}\right) d x^{1} d x^{2} \ldots d x^{m} .
$$

Применяя нётеровские граничные условия (13) и требуя, чтобы левая часть равенства (14) обращалась в нуль на многообразии решений, получаем "строгие" граничные условия [15]

$$
\left.R_{\alpha 1} L\right|_{x^{1} \rightarrow \partial D}=\left.R_{\alpha 2} L\right|_{x^{2} \rightarrow \partial D}=\cdots=\left.R_{\alpha m} L\right|_{x^{m} \rightarrow \partial D}=0 .
$$

Для $L=L\left(x, u, u_{i}\right)$ строгие граничные условия (15) принимают простой вид

$$
\left.\alpha^{a} \frac{\partial L}{\partial u_{l}^{a}}\right|_{x^{l} \rightarrow \partial D}=0, \quad l=1, \ldots, m
$$

В работе [15] было показано, что в случае произвольной функции независимых переменных условия (13) и (15), необходимые для существования нётеровских законов сохранения, допускают лишь конечное число существенных законов сохранения, соответствующих бесконечной симметрии системы.

2.2. Произвольные функции зависимых переменных. Теперь рассмотрим бесконечные симметрии, генераторы которых содержат произвольные гладкие функции $f$ от зависимых переменных и их производных:

$$
X_{\alpha} L=D_{i} M_{i}, \quad \alpha^{a}=f^{a}\left(u, u_{k}, \ldots\right), \quad i, k=1, \ldots, m+1, \quad a=1, \ldots, n .
$$

Закон сохранения (10) в этом случае имеет вид

$$
D_{l}\left(M_{l}-R_{\alpha l} L\right)+D_{t}\left(M_{t}-R_{\alpha t} L\right) \doteq 0, \quad l=1, \ldots, m,
$$


где

$$
\begin{aligned}
M_{i} & =A_{i} f\left(u, u_{k}, \ldots\right)+B_{i s} D_{s} f\left(u, u_{k}, \ldots\right)+\cdots, \\
R_{\alpha} L & =P_{i} f\left(u, u_{k}, \ldots\right)+Q_{i s} D_{s} f\left(u, u_{k}, \ldots\right)+\cdots,
\end{aligned}
$$

а индекс $s$ нумерует аргументы функции $f\left(u, u_{k}, \ldots\right)$.

Чтобы система обладала (нётеровскими) локальными сохраняющимися величинами, должны выполняться и нётеровские граничные условия (13), и строгие граничные условия (15). Пусть

$$
f\left(u, u_{k}, \ldots\right)=f\left(\xi\left(u, u_{k}, \ldots\right)\right)
$$

В предположении о регулярных граничных условиях на бесконечности

$$
u^{a} \underset{x \rightarrow \pm \infty}{\longrightarrow} 0, \quad u_{i}^{a} \underset{x \rightarrow \pm \infty}{\longrightarrow} 0, \quad i=1, \ldots, m+1, \quad a=1, \ldots, n,
$$

нётеровские граничные условия (13) принимают вид

$$
\begin{aligned}
& M_{i}\left(f(\xi(0, \ldots, 0)), f^{\prime}(\xi(0, \ldots, 0)), \ldots\right)- \\
& \quad-M_{i}\left(f(\xi(0, \ldots, 0)), f^{\prime}(\xi(0, \ldots, 0)), \ldots\right)=0, \quad i=1, \ldots, m+1 .
\end{aligned}
$$

Данные условия выполнены для любой гладкой функции $f$ при выполнении неравенства

$$
|\xi(0,0, \ldots, 0)|<\infty
$$

Легко видеть, что при данном ограничении строгие граничные условия (15) также выполнены.

Таким образом, в отличие от случая функций от независимых переменных, для бесконечных симметрий с произвольными функциями от зависимых переменных $\alpha^{a}=f^{a}\left(u, u_{k}, \ldots\right)$ нет серьезных ограничений для того, чтобы функции $f^{a}$ приводили к локальным законам сохранения; уравнение непрерывности (18) при этом дает бесконечное число существенных законов сохранения [25]. Соответствующие нётеровские сохраняющиеся величины можно найти из выражения

$$
D_{t} \int\left(M_{t}-R_{\alpha t} L\right) d x^{1} d x^{2} \ldots d x^{m} \doteq 0
$$

\section{3. ДИФФЕРЕНЦИАЛЬНЫЕ СИСТЕМЫ С БЕСКОНЕЧНЫМИ СИММЕТРИЯМИ}

В работе [25] нам удалось найти несколько классов скалярных дифференциальных уравнений в частных производных, обладающих бесконечными симметриями, с произвольными функциями от зависимых переменных и их производных, а также получить соответствующие бесконечные наборы существенных законов сохранения. Здесь мы рассмотрим сходную задачу для лагранжевых систем дифференциальных уравнений.

Будем искать системы с лагранжианами первого порядка

$$
L=L\left(u, v, u_{i}, v_{i}\right), \quad i=x, t, \quad u=u(x, t), \quad v=v(x, t),
$$


которые допускают бесконечные вариационные симметрии $X_{\alpha}(4)$ с произвольной функцией $f(\xi(u, v))$ от зависимых переменных $u, v$, а именно

$$
\begin{aligned}
& \alpha^{u}=p^{u} f(\xi)+q^{u} f^{\prime}(\xi)+r^{u} f^{\prime \prime}(\xi), \\
& \alpha^{v}=p^{u} f(\xi)+q^{v} f^{\prime}(\xi)+r^{v} f^{\prime \prime}(\xi),
\end{aligned}
$$

где $p^{u}, p^{v}, q^{u}, q^{v}, r^{u}, r^{v}$ суть функции от $x, t, u, v$.

В нашем случае

$$
X_{\alpha} L=\alpha^{u} L_{u}+\left(D_{x} \alpha^{u}\right) L_{z}+\left(D_{t} \alpha^{u}\right) L_{w}+\alpha^{v} L_{v}+\left(D_{x} \alpha^{v}\right) L_{\bar{z}}+\left(D_{t} \alpha^{v}\right) L_{\bar{w}}
$$

где $u_{x} \equiv z, u_{t} \equiv w, v_{x} \equiv \bar{z}, v_{t} \equiv \bar{w}$. Потребуем, чтобы $X_{\alpha}$ было вариационной симметрией,

$$
X_{\alpha} L=D_{x} M_{x}+D_{t} M_{t}
$$

где

$$
\begin{aligned}
M_{x} & =A f(\xi)+B f^{\prime}(\xi)+C f^{\prime \prime}(\xi), \\
M_{t} & =E f(\xi)+F f^{\prime}(\xi)+G f^{\prime \prime}(\xi),
\end{aligned}
$$

а коэффициенты $A, B, C, E, F, G$ суть функции от $x, t, u, v$. Воспользовавшись произвольностью функции $f(\xi)$, получим следующие связи:

$$
\begin{aligned}
\left(\xi_{u} z\right. & \left.+\xi_{v} \bar{z}\right)\left(r^{u} L_{z}+r^{v} L_{\bar{z}}-C\right)+\left(\xi_{u} w+\xi_{v} \bar{w}\right)\left(r^{u} L_{w}+r^{v} L_{\bar{w}}-G\right)=0 \\
\left(\xi_{u} z\right. & \left.+\xi_{v} \bar{z}\right)\left(q^{u} L_{z}+q^{v} L_{\bar{z}}-B\right)+\left(\xi_{u} w+\xi_{v} \bar{w}\right)\left(q^{u} L_{w}+q^{v} L_{\bar{w}}-F\right)+ \\
& +r^{u} L_{u}+r^{v} L_{v}+\left(r_{u}^{u} L_{z}+r_{u}^{v} L_{\bar{z}}-C_{u}\right) z+\left(r_{v}^{u} L_{z}+r_{v}^{v} L_{\bar{z}}-C_{v}\right) \bar{z}+ \\
& +\left(r_{u}^{u} L_{w}+r_{u}^{v} L_{\bar{w}}-G_{u}\right) w+\left(r_{v}^{u} L_{w}+r_{v}^{v} L_{\bar{w}}-G_{v}\right) \bar{w}=0 \\
\left(\xi_{u} z\right. & \left.+\xi_{v} \bar{z}\right)\left(p^{u} L_{z}+p^{v} L_{\bar{z}}-A\right)+\left(\xi_{u} w+\xi_{v} \bar{w}\right)\left(p^{u} L_{w}+p^{v} L_{\bar{w}}-E\right)+ \\
& +q^{u} L_{u}+q^{v} L_{v}+\left(D_{x} q^{u}\right) L_{z}+\left(D_{x} q^{v}\right) L_{\bar{z}}-D_{x} B+ \\
& +\left(D_{t} q^{u}\right) L_{w}+\left(D_{t} q^{v}\right) L_{\bar{w}}-D_{t} F=0 \\
p^{u} L_{u} & +p^{v} L_{v}+\left(D_{x} p^{u}\right) L_{z}+\left(D_{x} p^{v}\right) L_{\bar{z}}-D_{x} A+\left(D_{t} p^{u}\right) L_{w}+\left(D_{t} p^{v}\right) L_{\bar{w}}-D_{t} E=0
\end{aligned}
$$

Из уравнения (28) можно получить следующий вид лагранжиана в зависимости от значений величин $r^{u}, r^{v}, q^{u}, q^{v}$.

СлУчАй А. Пусть $r^{u}, r^{v} \neq 0$, тогда

$$
L=a z+b \bar{z}+c w+d \bar{w}+R+\psi\left(\frac{z}{r^{u}}-\frac{\bar{z}}{r^{v}}, \frac{w}{r^{u}}-\frac{\bar{w}}{r^{v}}, \frac{\xi_{u} z+\xi_{v} \bar{z}}{\xi_{u} w+\xi_{v} \bar{w}}\right)
$$

где

$$
a=a(u, v), \quad b=b(u, v), \quad c=c(u, v), \quad d=d(u, v), \quad R=R(u, v),
$$

функция $\psi$ произвольна и в общем случае $a d-b c \neq 0$. В специальном случае

$$
a=d=0, \quad b=-u^{2}, \quad c=v, \quad \psi=0, \quad R=\mu^{\prime \prime}(v)
$$


лагранжиан $L$ принимает вид

$$
L=v u_{t}-\frac{u^{2}}{2} v_{x}+\mu^{\prime \prime}(v) .
$$

Соответствующие уравнения движения записываются как

$$
v_{t}+u v_{x}=0, \quad u_{t}+u u_{x}+\mu^{\prime \prime \prime}(v)=0 .
$$

Эта система представляет собой систему уравнений Гуревича-Зыбина, описывающих формирование крупномасштабных структур во Вселенной [28].

Другой специальный случай задается условиями

$$
a(u, v)=K(u, v), \quad b=S(u, v), \quad c=0, \quad \psi=0, \quad d=R=0 .
$$

При этом

$$
L=u v_{t}+K(u, v) u_{x}+S(u, v) v_{x}
$$

а система уравнений движения имеет вид

$$
u_{t}=M(u, v) u_{x}, \quad v_{t}=M(u, v) v_{x}
$$

где $M=K_{v}-S_{u}$, и представляет собой систему гидродинамического типа (см., например, работу [29]).

Как известно, любые уравнения с лагранжианами (32) (включая (33) и (35)) имеют бесконечное число существенных законов сохранения вида (18). Это же заключение имеет место и для уравнений, рассмотренных далее в случаях Б и В.

СлУЧАй Б. Пусть $r^{v}=0, r^{u} \neq 0$, тогда

$$
L=z \frac{C}{r^{u}}+w \frac{G}{r^{u}}+R+\psi\left(\bar{z}, \bar{w}, \frac{\xi_{u} z+\xi_{v} \bar{z}}{\xi_{u} w+\xi_{v} \bar{w}}\right)
$$

где $C, G, R, \xi$ суть функции от $u, v$.

СлУчАй В. Пусть $r^{u}=0, r^{v} \neq 0$, тогда

$$
L=\bar{z} \frac{C}{r^{u}}+\bar{w} \frac{G}{r^{u}}+R+\psi\left(z, w, \frac{\xi_{u} z+\xi_{v} \bar{z}}{\xi_{u} w+\xi_{v} \bar{w}}\right),
$$

где $C, G, R, \xi$ - функции от $u, v$.

СлУчАЙ Г. Пусть $r^{u}=r^{v}=0$. Анализ этого случая сводится к нахождению дифференциальных систем, допускающих вариационные симметрии вида

$$
\alpha^{u}=p^{u} f(\xi)+q^{u} f^{\prime}(\xi), \quad \alpha^{v}=p^{u} f(\xi)+q^{v} f^{\prime}(\xi) .
$$

Аналогично нашим предыдущим результатам эти уравнения определяют широкий класс дифференциальных систем, обладающих бесконечным числом существенных законов сохранения (и бесконечными симметриями). 
СлУчАй Г1. Пусть $q^{u}, q^{v} \neq 0$, тогда

$$
L=a z+b \bar{z}+c w+d \bar{w}+R+\psi\left(\frac{z}{q^{u}}-\frac{\bar{z}}{q^{v}}, \frac{w}{q^{u}}-\frac{\bar{w}}{q^{v}}, \frac{\xi_{u} z+\xi_{v} \bar{z}}{\xi_{u} w+\xi_{v} \bar{w}}\right),
$$

где

$$
a=a(u, v), \quad b=b(u, v), \quad c=c(u, v), \quad d=d(u, v), \quad R=R(u, v) .
$$

СлУчАй Г2. Пусть $q^{v}=0, q^{u} \neq 0$, тогда

$$
L=z \frac{C}{q^{u}}+w \frac{G}{q^{u}}+R+\psi\left(\bar{z}, \bar{w}, \frac{\xi_{u} z+\xi_{v} \bar{z}}{\xi_{u} w+\xi_{v} \bar{w}}\right),
$$

где

$$
C=C(u, v), \quad G=G(u, v), \quad R=R(u, v), \quad \xi=\xi(u, v) .
$$

СлУчАй ГЗ. Пусть $q^{u}=0, q^{v} \neq 0$, тогда

$$
L=\bar{z} \frac{C}{q^{u}}+\bar{w} \frac{G}{q^{u}}+R+\psi\left(z, w, \frac{\xi_{u} z+\xi_{v} \bar{z}}{\xi_{u} w+\xi_{v} \bar{w}}\right) .
$$

Анализ случая $q^{v}=q^{u}=0$ приводит к похожим результатам.

Итак, в настоящей работе мы нашли большое число систем дифференциальных уравнений в частных производных, которые допускают алгебры бесконечных симметрий, содержащие произвольные функции от зависимых переменных и бесконечное число существенных законов сохранения. Заметим, что, в отличие от систем, рассмотренных в настоящей работе, алгебры симметрий уравнений типа уравнения Лиувилля содержат произвольные функции, зависящие от первой и второй производных зависимых переменных [21]. Для нахождения дифференциальных систем с бесконечным набором сохраняющихся плотностей мы могли бы воспользоваться тем, что нам известно большое число скалярных уравнений второго порядка, обладающих этим свойством, и далее преобразовать наше скалярное дифференциальное уравнение второго порядка в систему двух уравнений первого порядка. Мы приведем здесь только один пример; более подробный анализ будет представлен отдельно. Из работы [25] мы знаем, что любые лагранжианы вида $L=\psi\left(q_{x}, q_{t}\right)$ с произвольной функцией $\psi$ имеют бесконечные вариационные симметрии, содержащие произвольные функции от зависимых переменных, а потому и бесконечное число сохраняющихся плотностей. Если выбрать $L=q_{t}^{2} / 2-G\left(q_{x}\right)$, то соответствующие уравнения Эйлера-Лагранжа будут иметь вид $q_{t t}=-q_{x x} G^{\prime \prime}\left(q_{x}\right)$. Используя переменные $r=q_{x}$, $u=q_{t},\left(F(r)=-G^{\prime}(r)\right)$, получим систему уравнений

$$
u_{t}=D_{x}(F(r)), \quad r_{t}=u_{x},
$$

которая описывает политропный газ [30].

\section{4. ЗАКЛЮЧЕНИЕ}

Бесконечные симметрии с произвольными функциями от (не всех) независимых переменных приводят к конечному числу существенных законов сохранения. В отличие от этих симметрий бесконечные симметрии с произвольными функциями от

Теоретическая и математическая физика, т. 160, № 1, 2009 г. 
зависимых переменных приводят к бесконечному числу существенных законов сохранения. Этим свойством обладают разнообразные системы из двух дифференциальных уравнений первого порядка. Примерами таких систем являются хорошо известные уравнения гидродинамического типа, уравнение Гуревича-Зыбина, а также уравнения политропного газа. Естественным продолжением настоящей работы была бы попытка понять значение бесконечного числа локальных законов сохранения с произвольными функциями от зависимых переменных, а также связь с задачей линеаризации.

Благодарности. Я благодарен рецензенту за полезные предложения и М. Павлову за многочисленные стимулирующие обсуждения.

\section{Список литературы}

[1] E. Noether, Nachr. Akad. Wiss. Göttingen Math.-Phys. Kl., 1918, № 2, 235-257.

[2] П. Олвер, Приложения групп Ли к дифференциальным уравнениям, Мир, М., 1989.

[3] J. Hanc, S. Tuleja, M. Hancova, Amer. J. Phys., 72:4 (2004), 428-435.

[4] N. H. Ibragimov, T. Kolsrud, Nonlinear Dynam., 36:1 (2004), 29-40.

[5] S. C. Anco, G. Bluman, European J. Appl. Math., 13:5 (2002), 545-566; 567-585.

[6] T. Wolf, European J. Appl. Math., 13:2 (2002), 129-152.

[7] A. H. Kara, F. M. Mahomed, Internat. J. Theoret. Phys., 39:1 (2000), 23-40.

[8] I. M. Anderson, J. Pohjanpelto, Math. Proc. Cambridge Philos. Soc., 120:2 (1996), 369-384.

[9] А. Г. Мешков, Изв. вузов. Физика, 38:7 (1995), 9-14.

[10] V. Rosenhaus, G. H. Katzin, J. Math. Phys., 35:4 (1994), 1998-2012.

[11] A.M. Vinogradov (ed.), Symmetries of partial differential equations. Conservation Laws-Applications-Algorithms., Springer, Dordrecht, 1989.

[12] A. M. Vinogradov, Acta Appl. Math., 2:1 (1984), 21-78.

[13] R. Jackiw, N. S. Manton, Ann. Phys., 127:2 (1980), 257-273.

[14] N.H. Ibragimov (ed.), Lie Group Analysis of Differential Equations, V. 1-3, CRC, Boca Raton, 1994; 1995; 1996.

[15] V. Rosenhaus, J. Math. Phys., 43:12 (2002), 6129-6150.

[16] V. Rosenhaus, Rep. Math. Phys., 51:1 (2003), 71-86.

[17] V. Rosenhaus, J. Nonlinear Math. Phys., 13:2 (2006), 255-270.

[18] V. Rosenhaus, J. Phys. A, 39:24 (2006), 7693-7703.

[19] В. Розенхаус, ТМФ, 144:1 (2005), 190-198.

[20] А. В. Жибер, А. Б. Шабат, Докл. АН СССР, 247:5 (1979), 1103-1107.

[21] А. В. Жибер, Н. Х. Ибрагимов, А. Б. Шабат, Докл. АН ССCP, 249:1 (1979), 26-29.

[22] А. В. Жибер, В. В. Соколов, УМН, 56:1(337) (2001), 63-106.

[23] V. V. Sokolov, A. V. Zhiber, Phys. Lett. A, 208:4-6 (1995), 303-308.

[24] I. M. Anderson, M. Juráš, Duke Math. J., 89:2 (1997), 351-375.

[25] В. Розенхаус, ТМФ, 151:3 (2007), 518-528.

[26] В. В. Жаринов, "Low-dimensional conservation laws of PDE", Избранные вопросъ математической физики и анализа, Тр. МИАН, 203, 1994, 478-493.

[27] J. Rosen, Some Properties of the Euler-Lagrange Operators, Preprint TAUP-269-72, Tel Aviv Univ., Tel Aviv, 1972.

[28] M. V. Pavlov, J. Phys. A, 38:17 (2005), 3823-3840.

[29] М. В. Павлов, ТМФ, 150:2 (2007), 263-285.

[30] A. Das, Z. Popowicz, Phys. Lett. A, 296:1 (2002), 15-26. 